\title{
Dynamic Stall Control by Passive Disturbance Generators
}

\author{
B. Heine*, K. Mulleners ${ }^{\dagger}$ \\ DLR Göttingen AS-HE, Göttingen, Germany \\ G. Joubert ${ }^{\ddagger}$ \\ ONERA DAAP/H2T, Meudon, France \\ and M. Raffel ${ }^{\S}$ \\ DLR Göttingen AS-HE, Göttingen, Germany
}

\begin{abstract}
Passive low-aspect ratio cylinders mounted near the leading edge of an airfoil have been found to significantly improve the performance under dynamic stall conditions. In order to clarify the principle of operation these cylindrical disturbance generators (DG), high speed PIV and simultaneous pressure measurements have been performed on the pitching rotary aircraft wing profile OA209. Time resolved information for the flow field at mid chord and the pressure distribution was taken. In addition to the best configuration found in previous experiments, various geometries and sizes of these devices have been investigated. The experiments showed the effectiveness of the disturbance generators in reducing the negative pitching moment peak and hysteresis effects. Moreover, the development of stall compared to the clean case has been investigated and it was found that the disturbance generators reduce the strength and the distance normal to the chord of the vortices.
\end{abstract}

\section{Nomenclature}

\begin{tabular}{|c|c|c|}
\hline$a_{n}$ & & POD coefficient \\
\hline$b$ & {$[\mathrm{~m}]$} & Span \\
\hline$c$ & {$[\mathrm{~m}]$} & Chord length \\
\hline$c_{l}$ & & Lift coefficient \\
\hline$c_{m}$ & & Pitching moment coefficient \\
\hline$D$ & & Diameter or length of disturbance generator \\
\hline$f_{\alpha}$ & {$[\mathrm{Hz}]$} & Pitching frequency \\
\hline$h$ & {$[\mathrm{~m}]$} & Height of the disturbance generator \\
\hline$h_{t r}$ & {$[\mathrm{~m}]$} & Transition strip height \\
\hline$k$ & & Reduced frequency, $\omega c / 2 U_{\infty}$ \\
\hline $\mathrm{M}$ & & Mach number \\
\hline Re & & Chord Reynolds number \\
\hline$s$ & {$[\mathrm{~m}]$} & Spacing of the disturbance generator \\
\hline$U_{\infty}$ & {$\left[\frac{\mathrm{m}}{\mathrm{s}}\right]$} & Free stream velocity \\
\hline$\alpha$ & {$\left[{ }^{\circ}\right]$} & Angle of attack \\
\hline$\alpha_{0}$ & {$\left[{ }^{\circ}\right]$} & Mean angle of attack \\
\hline$\hat{\alpha}$ & {$\left[{ }^{\circ}\right]$} & Pitching amplitude \\
\hline
\end{tabular}

\footnotetext{
*PhD Student, German Aerospace Center, Dept. Helicopters, Bunsenstrasse 10, 37073 Göttingen

${ }^{\dagger}$ Postdoc, German Aerospace Center, Dept. Helicopters, Bunsenstrasse 10, 37073 Göttingen

${ }^{\ddagger}$ PhD Student, ONERA, DAAP/H2T, 8 rue des Vertugadins, 92190 Meudon

§Professor, German Aerospace Center, Dept. Helicopters, Bunsenstrasse 10, 37073 Göttingen
} 


$\begin{array}{lll}\delta & {[\mathrm{m}]} & \text { Boundary layer thickness } \\ \delta^{*} & {[\mathrm{~m}]} & \text { Displacement thickness } \\ \Delta S_{n}^{*} & & \text { Damping coefficients for n/ref } \\ \Lambda & & \text { Aspect ratio } \\ \psi & {[1 / \mathrm{s}]} & \text { y-vorticity }\end{array}$

\section{Introduction}

$\mathrm{T}$

HE high speed characteristics of rotary wing aircraft are limited by two factors: transonic flow at the tip of the advancing blade and stall at the retreating blade. While at the advancing blade rotational and forward speeds add, the retreating blade faces low velocities and even reverse flow. To maintain trimmed lift over the rotor cycle, high angles of attack are necessary for the retreating blade. The rotor blade therefore has to perform a pitching motion. At a high forward speed, stall occurs on parts of the retreating blade. Compared to the static stall case, maximum lift and stall angle are delayed due to the dynamic pitching movement of the blade. This phenomenon, referred to as dynamic stall, leads to a series of complex aerodynamic mechanisms and has been studied by numerous researchers. ${ }^{1-3}$ In most cases dynamic stall induces an abrupt decay and fluctuation of the aerodynamic loads, resulting in high structural loads, drag and control forces. The pitching moments can cause aeroelastic flutter with negative damping. Dynamic stall is therefore considered to be a limiting factor for the helicopter's forward flight and maneuver performance, but also for the aeroelastic stability of e.g. wind turbines.

McCroskey ${ }^{1}$ and Carr et $a .^{2}{ }^{2}$ performed comprehensive studies on the phenomenon of dynamic stall and found a concentrated spanwise vortex as the dominant flow structure. For airfoils with leading edge stall, a strong vortex is formed close to the leading edge of the airfoil and travels downstream over the airfoil after the dynamic stall angle is reached. During its convective downstream motion, the vortex temporarily induces high lift and often strong negative pitching moments. Counter-rotating vortices from the trailing edge cause the dynamic stall vortex to depart from the airfoil's surface and the flow on the suction side is completely separated. ${ }^{4}$ The following downstroke movement delays the reattachment of the flow, leading to a large hysteresis. Finally, the boundary layer generally attaches from front to rear and the aerodynamic coefficients return to unstalled values.

Numerous passive and active dynamic stall control devices such as droop nose, ${ }^{5}$ model deformation, ${ }^{6}$ leading edge slat, ${ }^{7,8}$ periodic excitation ${ }^{9}$ or plasma actuators ${ }^{10}$ have been developed and investigated throughout the last decades. The aim of such control devices is i) to reduce the negative pitch moment peak, ii) to maintain or reduce drag and iii) to maintain or increase lift during one oscillation. Martin et al. ${ }^{11}$ applied counter rotating vane type vortex generators on a pitching VR7 airfoil at Mach numbers $0.3 \leq M \leq 0.4$ for various pitching frequencies and amplitudes. The vortex generators were found to have positive effects at lower Mach numbers up to $M=0.3$ at light stall conditions in terms of maximum lift and in reducing the pitching moment peak. However, they failed for higher Mach numbers and deep dynamic stall cases.

Geissler et al. ${ }^{12}$ developed a very simple, retro-fit capable passive device that improved the aerodynamic performance of a wing under dynamic stall conditions significantly, and only had minor influence on the rest of the cycle. The final dimension of the cylindrical $L E V o G s^{13,14}$ (Leading Edge Vortex Generators) was found after a parameter study where spacing, height and diameter were optimized. ${ }^{15}$ The best results were achieved using these DGs with a height of $0.0018 \mathrm{c}$ and a diameter of $0.02 \mathrm{c}$. The DGs were spaced equidistantly at $0.067 \mathrm{c}$ in the spanwise direction, slightly below the leading edge of the airfoil. The DGs were made from self-adhesive rubber and were simply glued onto an existing wing or blade. In figure 1 a

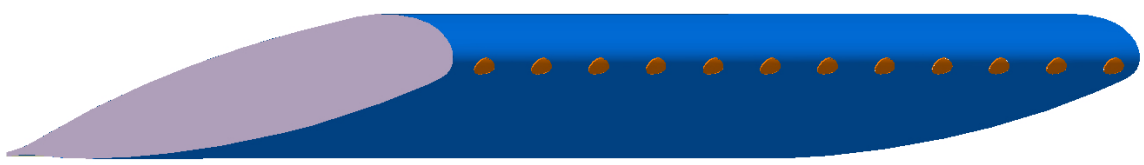

Figure 1. Drawing of the OA209 airfoil with LEVoGs.

drawing of the OA209 airfoil with LEVoGs is shown. The DGs are positioned in the stagnation region for 
moderate angles of attack, such that the influence of the DGs on the flow field is negligible for low angles of attack. However, as the angle of attack increases, the stagnation line moves downstream on the lower side of the airfoil. As a consequence, the DGs become active as they are exposed to flow coming forward from the stagnation line and around the leading edge of the airfoil.

Mai et al. ${ }^{15}$ assumed that the DGs induce streamwise vortices at high angles of attack that entrain high momentum free stream fluid towards the airfoil's surface such, that separation is delayed. In the onset of stall they also found a dominant three-dimensional effect on the upper surface where the individual wakes of the DGs bundle and eventually separate together.

Wind and water tunnel experiments on an OA209 airfoil by Heine et al. ${ }^{16}$ showed the significant global impact of the DGs on the flow which using PIV and dye flow visualization. Since these experiments on static and dynamically pitching airfoils could not satisfactorily clarify the principle of operation of the DGs, more fundamental experiments were performed on a circular cylinder by Heine et al. ${ }^{17}$ The investigations showed that, similar to the airfoil experiment by Mai et al., the wakes of the vortex generators bundle depending on the position of the DGs. This effect is highly unsteady and the wakes rapidly regroup and move in spanwise direction. It is expected that the high unsteadiness and three-dimensionality of this bundling effect reduces the strong coherent structure of the dynamic stall vortex, and hence reduce the strong negative pitching moments and drag.

Further, the wake of a large scale LEVoG on a flat plate was investigated by means of planar and tomographic PIV. ${ }^{18}$ The wake of such cylinders differs greatly from cylinders with larger aspect ratios and instead of a complete vortex system, a simple pair of counter-rotating trailing vortices was found for device heights well above the boundary layer height. However, these vortices decay quickly and have lost already $90 \%$ of their circulation at one diameter downstream. This is in agreement with the previous investigations, where no pronounced longitudinal vortices have been found further downstream of the LEVoG. The DGs might therefore rather be called disturbance generators (DG) than vortex generators.

Even though the previous experiments proved the capabilities of DGs and several useful observations have been made, the principle of operation is still not fully understood. This however, is an important task in order to further optimize such devices and adapt them to different situations.

The aim of this paper is to examine the principle of operation of leading edge disturbance generators. This was done by varying several parameters related to dynamic stall such as the mean angle of attack $\alpha_{0}$, the amplitude $\hat{\alpha}$ and the pitching frequency $f_{\alpha}$. In order to capture the consecutive events in the flow field above the airfoil, time resolved particle image velocimetry with simultaneous surface pressure measurements were employed. While Mai et al. investigated only circular shapes of disturbance generators, the present investigations were expanded towards different geometries in order to study the influence of the shape on the dynamic stall vortex and the efficiency of the airfoil. Further, the influence of reduced size and increased spacing of the disturbance generators was observed. This leads to a database with nine different types of motion for 12 different leading edge device configurations. Analyzed with post-processing tools such as the proper orthogonal decomposition method or vortex tracking algorithms, a detailed view on the principle of operation of disturbance generators is gained.

\section{Experimental Setup}

\section{II.A. Wind Tunnel and Airfoil Model}

The experiments were conducted in the F2 wind tunnel at the French Aerospace Lab ONERA in FaugaMauzac which has a closed test section with dimensions $1.8 \mathrm{~m} \times 1.4 \mathrm{~m} \times 5 \mathrm{~m}$ ( $\mathrm{W} \times \mathrm{H} \times \mathrm{L})$. The tunnel has a contraction ratio of 12 allowing a free stream velocity up to $U_{\infty}=55 \mathrm{~m} / \mathrm{s}$ with a turbulence level below $0.05 \%$. The free stream Reynolds number for all measurements was $\mathrm{Re}=1.8 \times 10^{6}$ based on the chord length $c$ and the Mach number was $\mathrm{M}=0.16$. A two-dimensional airfoil model with an OA209 profile with span $b=1.4 \mathrm{~m}$ and chord length $c=0.5 \mathrm{~m}$, yielding an aspect ratio $\Lambda=2.8$, was mounted onto a hydraulic pitching test rig and subjected to a sinusoidal oscillating motion about its quarter chord axis (for more details see Le Pape $e t$ $\left.a l .{ }^{19}\right)$. The mean incidence $\alpha_{0}$, amplitude $\hat{\alpha}$ and reduced frequency $k$, defined as $k=\pi f_{\alpha} c / U_{\infty}$ with $f_{\alpha}$ the oscillation frequency were varied, such that various light and deep dynamic stall conditions could be investigated (see Table 1). Since the chord-based Reynolds number was of the same order of magnitude as the full scale case, no transition tripping was applied for most measurements. The influence of tripping on the aerodynamic coefficients and the behavior of the disturbance generators was measured for several cases. 


\begin{tabular}{|c|c|c|c|c|}
\hline case & $\alpha_{0}$ & $\hat{\alpha}$ & $\mathbf{k}$ & $f_{\alpha}$ \\
\hline & {$\left[^{\circ}\right]$} & {$\left[^{\circ}\right]$} & & {$[\mathrm{Hz}]$} \\
\hline \hline I & 13 & 5 & 0.04 & 1.4 \\
\hline II & 13 & 5 & 0.1 & 3.5 \\
\hline III & 13 & 5 & 0.15 & 5.25 \\
\hline IV & 13 & 7 & 0.04 & 1.4 \\
\hline V & 13 & 7 & 0.1 & 3.5 \\
\hline VI & 13 & 7 & 0.15 & 5.25 \\
\hline VII & 15 & 5 & 0.04 & 1.4 \\
\hline VIII & 15 & 5 & 0.1 & 3.5 \\
\hline IX & 15 & 5 & 0.15 & 5.25 \\
\hline
\end{tabular}

Table 1. Parameter variation for the dynamic measurements $\left(\alpha=\alpha_{0}+\hat{\alpha} \sin \left(2 \pi f_{\alpha} t\right)\right)$.

\section{II.B. Measurement Methods}

Time-resolved PIV measurements were conducted in the cross sectional plane at model mid-span. In order to achieve both, high spatial and temporal resolution while covering the flow field over the entire chord length, four CMOS cameras were used simultaneously. Two cameras were placed in a stereoscopic configuration focussed on the leading edge region (cf. FOV Camera 1+2 in figure 2). Two cameras recorded the flow in the central and the trailing edge region (cf. FOV Camera 3 and FOV Camera 4 in figure 2). Illumination was provided by two double cavity diode pumped Nd:YLF lasers $(\lambda=527 \mathrm{~nm})$, each with a pulse energy of $30 \mathrm{~mJ}$ per pulse at $1000 \mathrm{~Hz}$ and a maximum frequency of $20 \mathrm{kHz}$. Time series of 10000 frames were recorded at $2100 \mathrm{~Hz}$, corresponding to an acquisition rate of $1050 \mathrm{~Hz}$ for the velocity fields. The time-resolved PIV data was evaluated according to standard procedures. ${ }^{20} \mathrm{~A}$ multi grid algorithm with image deformation was adopted within the correlation analysis and cross-correlation windows of $32 \times 32 \mathrm{px}$ with an overlap of $50 \%$ were used. The corresponding physical resolution was $0.0047 \mathrm{c}$. This procedure gave 750 velocity fields per cycle for a reduced frequency of $k=0.04$. Between two recordings, the free stream flow propagated $0.104 \mathrm{c}$. The measured velocity fields in the different fields of view were combined and then rotated into the airfoil reference system with the x-axis along the chord, the y-axis along the span and the z-axis upward perpendicular to the chord. The origin coincides with the rotation axis, i.e. the airfoils quarter chord axis, at model mid-span. The surface pressure distribution at the model mid-span was synchronized with the TR-

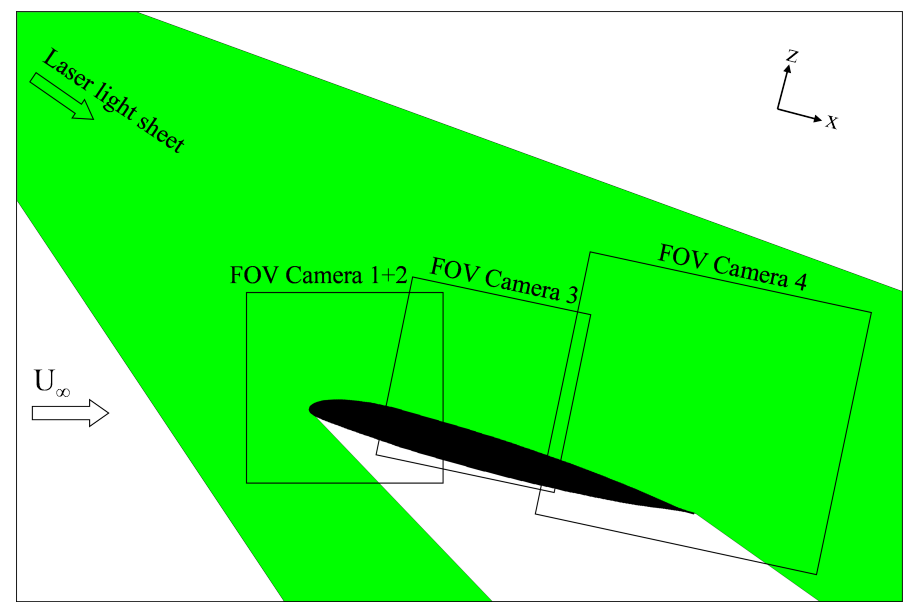

Figure 2. Positions of the PIV fields of view (FOV).

PIV and acquired at $25.6 \mathrm{kHz}$ during $8 \mathrm{~s}$ by 37 Kulite differential dynamic pressure transducers distributed chordwise on the pressure and suction side. The density of the transducers was increased in the leading 
edge region where high pressure gradients are expected. An integration of the pressure data allowed the determination of lift, pitching moments and the pressure drag. For the pressure measurements the sampling frequency was $2048 \mathrm{~Hz}$ with an acquisition time $t$ of $30 \mathrm{~s}$.

\section{II.C. Disturbance Generator Configurations}

The reference case discussed here concerns circular shaped DGs where aspect ratio, height and spacing correspond to Mai's ${ }^{15}$ 'best' configuration scaled with respect to the Reynolds number, yielding a height $h=0.18 \% \mathrm{c}$, a spacing $s=6.66 \% \mathrm{c}$, and an aspect ratio $\Lambda=$ $h / l=0.09$. The shape, dimensions and spacing of the disturbance generators were varied while keeping the aspect ratio constant. Table
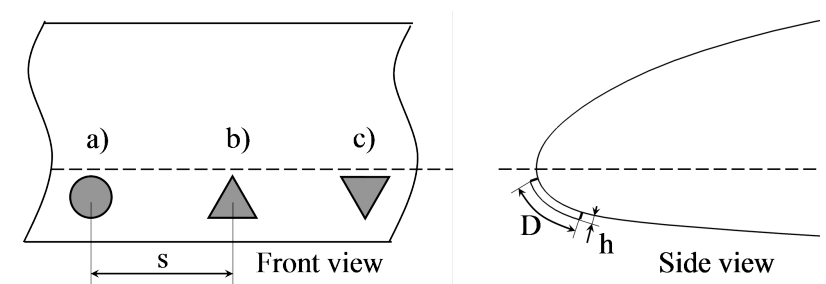

Figure 3. Sketch of the used DG shapes. a) circular b) backward wedge c) forward wedge. 2 summarizes the different disturbance generator configurations that were investigated. The different shapes (see also figure 3) that were considered are i) circular cylinders ii) forward equilateral wedges $(\triangle)$ and iii) backward equilateral wedges $(\nabla)$. The center of all disturbance generators was placed in the position of the stagnation line for $\alpha=10^{\circ}$. Special care was taken to ensure that the center pressure taps were located between two devices.

\begin{tabular}{|c|c|c|c|c|}
\hline No & Shape & Diameter or Length & Height & Spacing \\
\hline & & {$[\% \mathrm{c}]$} & {$[\% \mathrm{c}]$} & {$[\% \mathrm{c}]$} \\
\hline \hline 1 & $\bigcirc$ & 2 & 0.18 & 6.66 \\
\hline 2 & $\bigcirc$ & 2 & 0.18 & 10 \\
\hline 3 & $\bigcirc$ & 1.2 & 0.108 & 4 \\
\hline 4 & $\bigcirc$ & 1.2 & 0.108 & 6.66 \\
\hline 5 & $\triangle$ & 2 & 0.18 & 6.66 \\
\hline 6 & $\triangle$ & 2 & 0.18 & 10 \\
\hline 7 & $\triangle$ & 1.2 & 0.108 & 4 \\
\hline 8 & $\triangle$ & 1.2 & 0.108 & 6.66 \\
\hline 9 & $\nabla$ & 2 & 0.18 & 6.66 \\
\hline 10 & $\nabla$ & 2 & 0.18 & 4 \\
\hline 11 & $\nabla$ & 1.2 & 0.108 & 4 \\
\hline 12 & $\nabla$ & 1.2 & 0.108 & 6.66 \\
\hline
\end{tabular}

Table 2. All tested LEVoG configurations.

\section{Results and Discussion}

This study provides time-resolved velocity field information in addition to unsteady surface pressure distributions, allowing for direct correlation of fluctuations of the airloads with coherent or vortical structures in the flow field.

\section{III.A. Influence of Disturbance Generators on the Stall Development}

The influence of the DG on the aerodynamic loads during a single dynamic stall life cycle was investigated based on the flow field. In this section, we discuss this influence considering the reference DG case (circular shape with a height $h=0.18 \% \mathrm{c}$ and spacing $s=6.66 \% \mathrm{c}$ as presented in section II.C.) for the dynamic stall case IV ( $\alpha=13 \pm 7, k=0.04)$. 

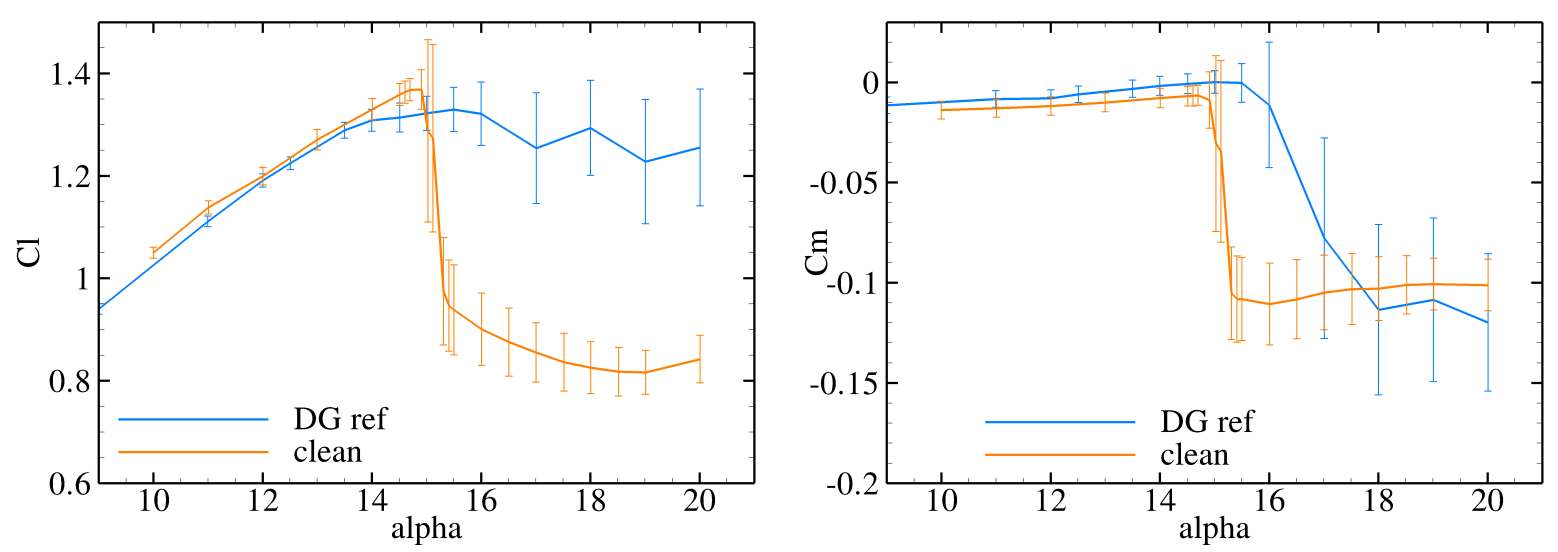

Figure 4. Lift coefficient and moment coefficient over angle of attack for the clean and DG case.
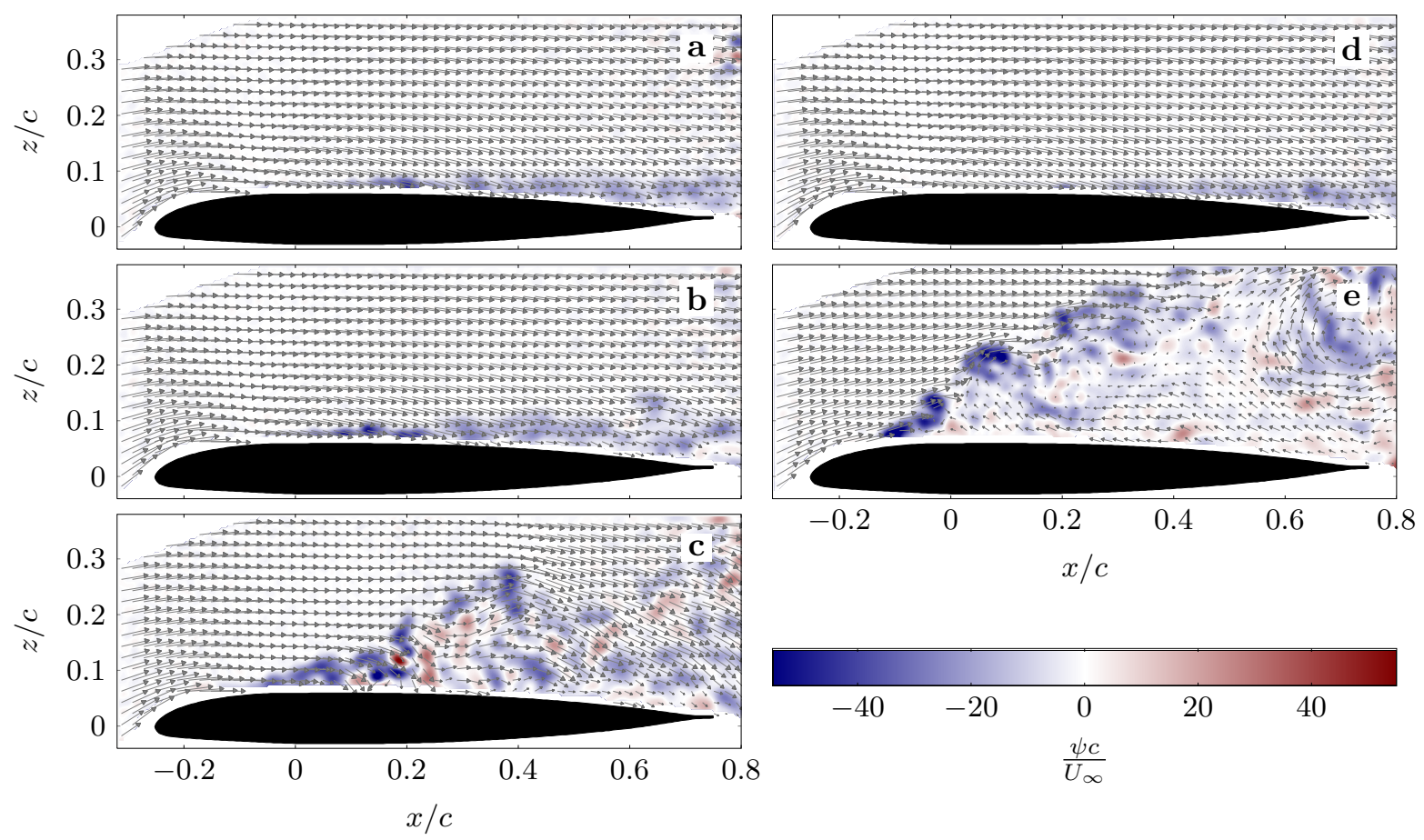

Figure 5. Unsteady velocity fields. Left column: DG reference at a) $\alpha=15^{\circ}$, b) $\alpha=16^{\circ}$, c) $\alpha=18^{\circ}$. Right column: clean at d) $\alpha=15^{\circ}$, e) $\alpha=15.5^{\circ}$. Color coded non-dimensionalized vorticity $\psi$.

\section{III.A.1. Static Stall}

For the static case, the DGs have a clear influence on the pressure distribution and hence on the lift and moment coefficients in figure 4 . Without the use of DG, lift stall occurs just after $\alpha=15^{\circ}$ and lift and moment coefficients drop abruptly. Three degrees before stall of the clean airfoil, the lift slope of the DG case is reduced. This is a consequence small disturbances near the wall that cause slightly earlier flow separation compared to the clean case (figure 5 a) and d))

If the angle of attack of the clean airfoil exceeds the maximum lift angle, separation point moves within $\Delta \alpha=0.5^{\circ}$ from the trailing edge to the leading edge (leading edge stall), causing an abrupt decay of lift (figure $5 \mathrm{~d}$ )-e)).

For the DG case, as the angle of attack is increased further, the separation point moves upstream slowly but stays attached for some portion of the airfoil (figure 5a)-c)) which is typical for airfoils with trailing edge stall. The loss of lift due to the separation is only slightly lower than the lift generated by the growing suction peak, resulting in lift coefficients that decrease only slowly.

It should be noted that for the clean case, fluctuations in the coefficients are high in the stall region and then are reduced again, while the fluctuations in the DG case remain at a constant high level if the 
maximum lift is exceeded. The lift rms values in the post stall region of the clean airfoil are approximately $100 \%$ higher for the DG case. An analysis of the velocity fields on different pressure ports reveal the origin of these strong fluctuations. The separation point shows a strong movement with respect to time, resulting in large differences in the pressure distribution and highly unsteady flow characteristics.

\section{III.A.2. Dynamic Stall}

A large influence of the DG compared to the clean case was found for the deep dynamic stall case $\alpha=13 \pm 7^{\circ}$, $k=0.04$ (figure 6). Considering dynamic stall, the influence of the DG on the lift and moment hysteresis curves is characterized by:

- Lower negative pitching moment peak

- Higher lift during downstroke

- Reduced hysteresis
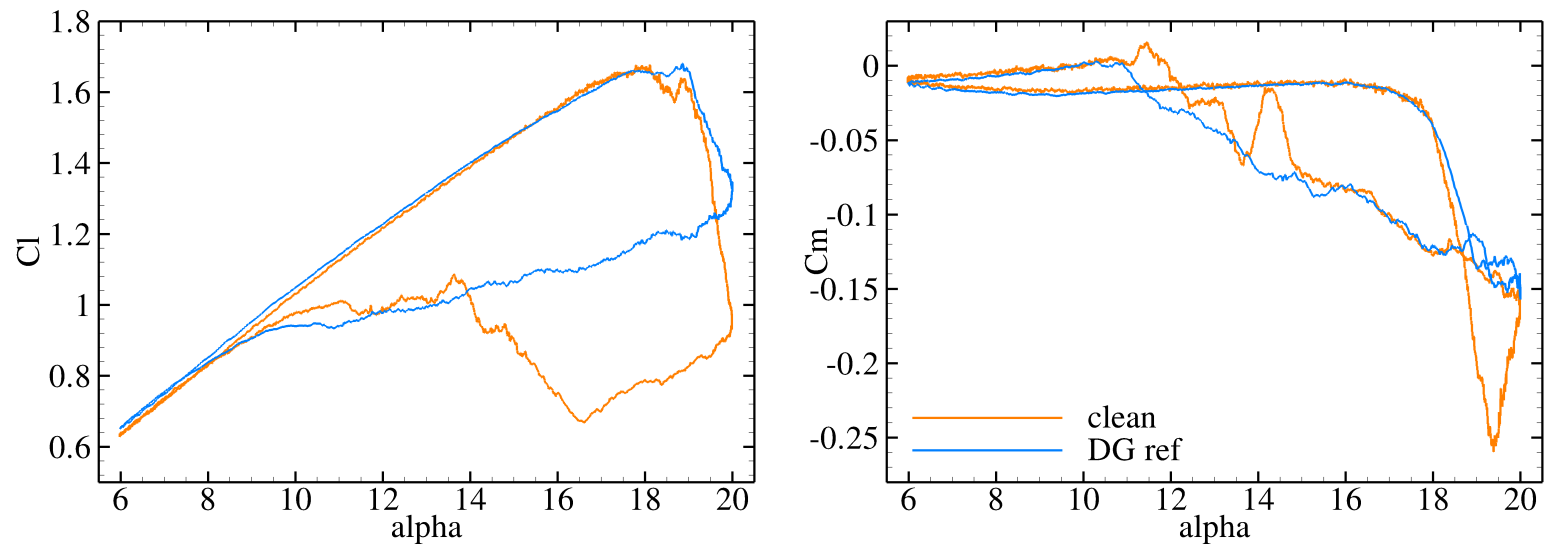

Figure 6. Time averaged dynamic lift and moment curves from pressure measurements plotted over angle of attack for clean and reference DG at $\alpha=13 \pm 7^{\circ}, \mathbf{k}=\mathbf{0 . 0 4}$
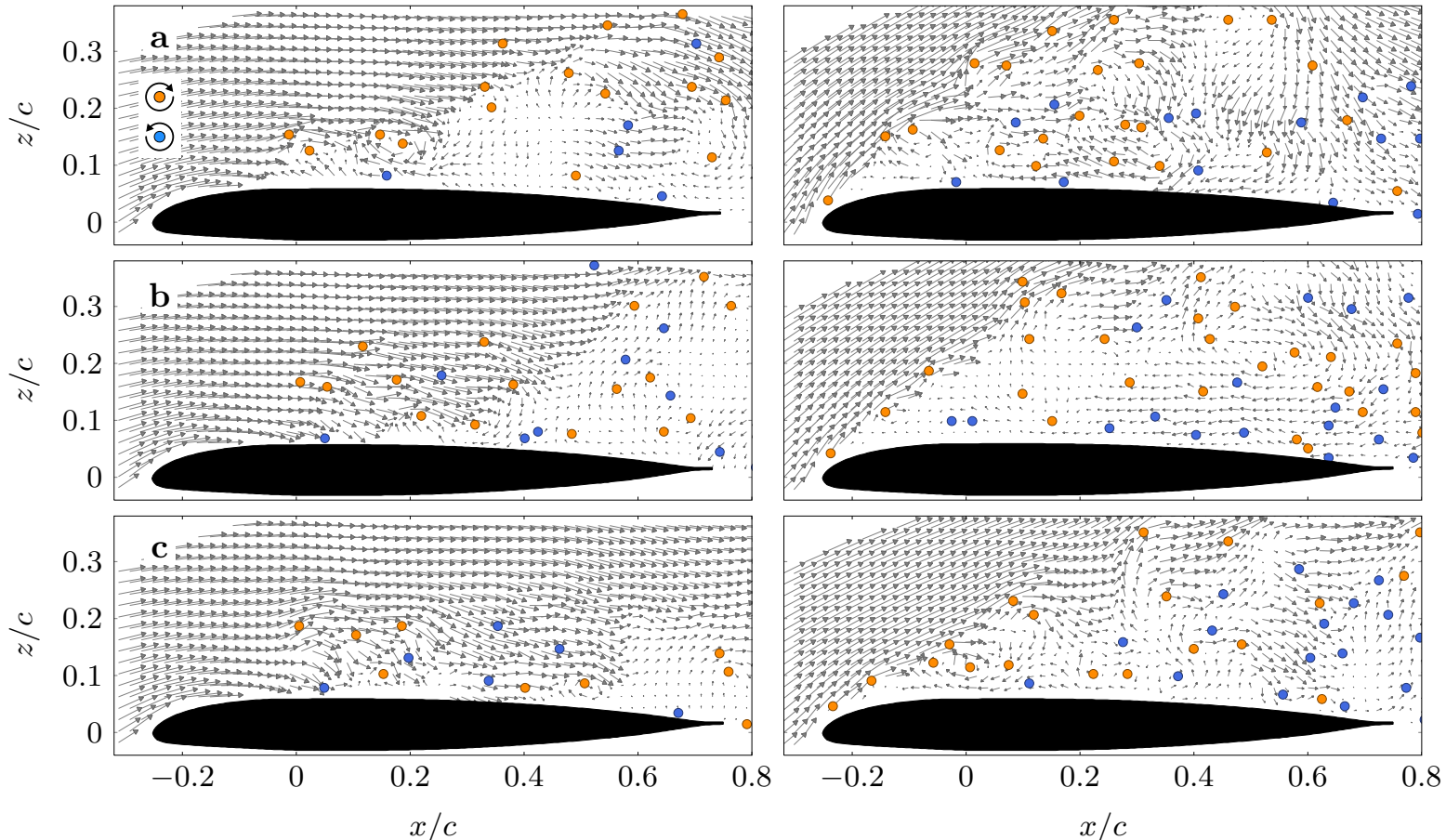

Figure 7. Instantaneous vector fields with $\Gamma_{2}$ vortex core detection at a) $\alpha=19.7^{\circ} \uparrow$, b) $\alpha=19.7^{\circ} \downarrow$, c) $\alpha=19.7^{\circ} \downarrow$ at $\alpha=13 \pm 7^{\circ}, \mathbf{k}=\mathbf{0 . 0 4}$ motion. Left column: reference $\mathbf{D G}$, right column: clean. 
The origin of these improvements can be found by analyzing the instantaneous vector fields. Figure 7 shows three vector fields for each case $\Delta \alpha=0.2^{\circ}$ below the maximum angle of attack. Vortex cores have been detected using the $\Gamma_{2}$ method $^{21,22}$ and are marked according to their sense of rotation.

In the present case, the DGs are capable of reducing the negative pitching moment peak by $50 \%$. This is a consequence of delayed flow separation of the DG case compared to the clean configuration (figure 7 a)-c)). Similar to the static case, sudden leading edge stall is present for the clean case, leading to a sharp decrease in the moment coefficient. In contrast, flow separation moves upstream more slowly for the DG case and stays attached in the first $20 \%$ chord, resulting in a smoother moment stall with a smaller peak in the pitching moment. Furthermore, the separated flow does not part as far from the upper surface as in the clean case, causing higher lift and facilitating reattachment.

The same effect explains the $60 \%$ higher lift at $\alpha=16.5^{\circ} \downarrow$. While the clean airfoil still suffers from complete separation on the upper side of the airfoil, the flow of the DG case starts to reattach starting at the leading edge. In general, this reduction of flow separation reduces the hysteresis loop. This observation is corroborated by the trajectories of the vortex cores for different phase intervals of a single dynamic stall cycle (figure 8) detected using the method of Mulleners et al. ${ }^{22}$ During upstroke $\left(13^{\circ} \leq \alpha \leq 16.5^{\circ}\right)$, the vortex trajectories of the clean and the DG cases show similar traces of the vortex cores. At the end of the upstroke $\left(16.5^{\circ} \leq \alpha \leq 20^{\circ}\right)$ the vortex cores of the clean case separate directly at the leading edge (compare also figure 7) and convect high above the airfoil's surface. For the DG, the vortices are formed further downstream and do not extend as high as for the clean case. If the angle of attack is reduced further (figure $8 \mathrm{c}$ )-d)), the vortex trajectories of the clean case remain qualitatively unchanged, while they indicate reattachment starting at the leading edge for the DG case.

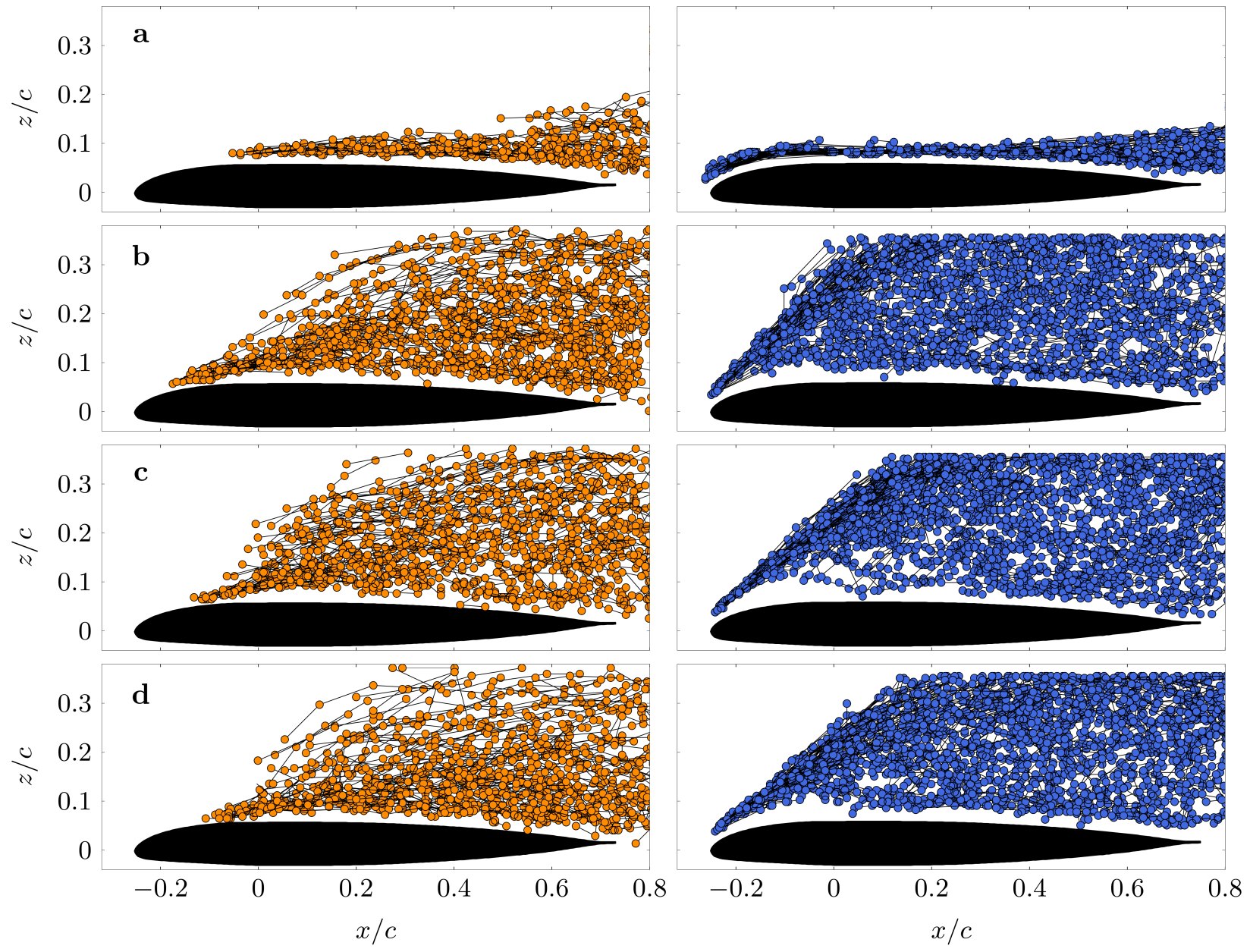

Figure 8. Vortex core trajectories for the clean (right column) and reference DG (left column) at $\alpha=13 \pm 7^{\circ}, \mathbf{k}=0.04$. a) $13^{\circ} \leq \alpha \leq 16.5^{\circ}$, b) $16.5^{\circ} \leq \alpha \leq 20^{\circ}$, c) $20^{\circ} \leq \alpha \leq 16.5^{\circ}$, d) $16.5^{\circ} \leq \alpha \leq 13^{\circ}$. 
The height distribution of the vortex cores over one cycle can be displayed if the non-dimensionalized distance between the airfoil chord is plotted over the angle of attack (figure 9). The vortex cores of the clean case leave the proximity of the airfoil abruptly at $\alpha \approx 16^{\circ} \uparrow$ (onset of moment stall), stay at a distance of $\Delta z / c \approx 0.17$ until $\alpha=14^{\circ} \downarrow$ and then quickly move close to the surface again (reattachment). For the reference DG case, the vortex cores are initially higher above the airfoil and increase height at the same angle as the clean case but stay closer to the wall. The differences between the individual vortices are high for the reference DG case, but at the maximum angle of attack the average height is about $25 \%$ lower than for the clean case.

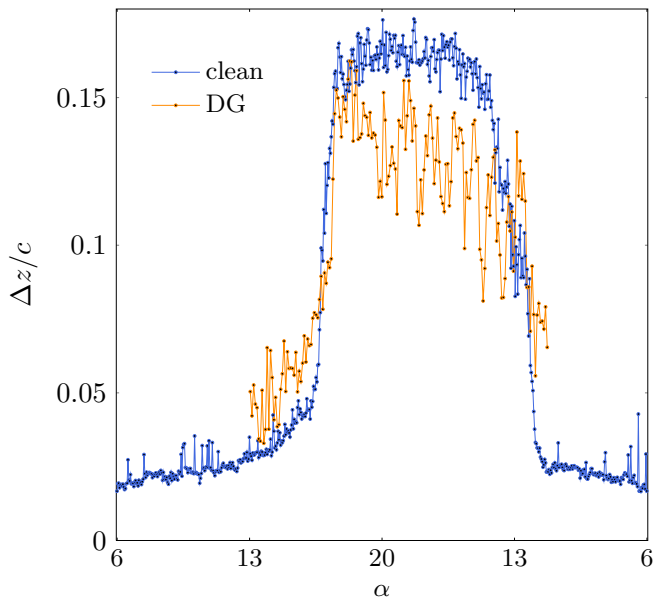

Figure 9. Averaged vortex core height distribution over one cycle for the clean and reference DG case at $\alpha=13 \pm 7^{\circ}$, $\mathrm{k}=0.04$.

In order to investigate the influence of the DGs on coherent structures a Proper Orthogonal Decomposition $(\mathrm{POD})^{23}$ has been applied to the time dependent vector fields in order to detect large scale coherent structures such as dynamic stall vortices. The vector fields are used to form an eigenvalue problem that is solved to find a set of basis functions, representing the flow field. Each eigenvalue reflects a fraction of the overall energy in the input vector fields, represented by the corresponding eigenfunction. Usually the method is applied to static or phase averaged cases, where the first mode represents the mean flow and the following modes the further structures in the flow field, sorted by energy content. In the present case, all images have been rotated in order to have the airfoil always at the same position of the image. Then, the POD has been applied to all images. The resulting eigenmodes can be interpreted as follows for the current test case: 22,24

- 1st mode: The most energetic structure represents the attached flow.

- 2nd mode: The separated flow

- 3rd mode: Strongest coherent structure besides mode 1 and 2 (dynamic stall vortex)

- 4 th $\leq$ mode $\leq \mathrm{n}$ : Weaker coherent structures up to turbulence and noise

The first three POD eigenvectors were calculated from 5000 images capturing 6.6 periods for the clean case are shown in the left column in figure 10. The first two eigenmodes represent the mean flow in the attached (first row in figure 10) and separated state (second row in figure 10). The eigenvector in the third row represents the most energetic coherent structure besides the mean flow which can be interpreted as the dynamic stall vortex in the present case. In the right column, the corresponding POD coefficients are plotted over the angle of attack for one cycle. For better comparison, the coefficients of the DG case have been projected onto the velocity field of the clean case. For all modes, the coefficients are close to zero when attached flow is present. At the onset of stall, the coefficients for the clean case rise abruptly, indicating suddenly occurring strong coherent structures in the flow. In contrast, the coefficients for the DG reference case increase earlier and more gradually, reaching their maximum approximately $\Delta \alpha \approx 1^{\circ}$ later than the clean case. Especially for the first two modes, the strength of the coherent structures is much lower for the DG case, indicating weaker coherent structures. 

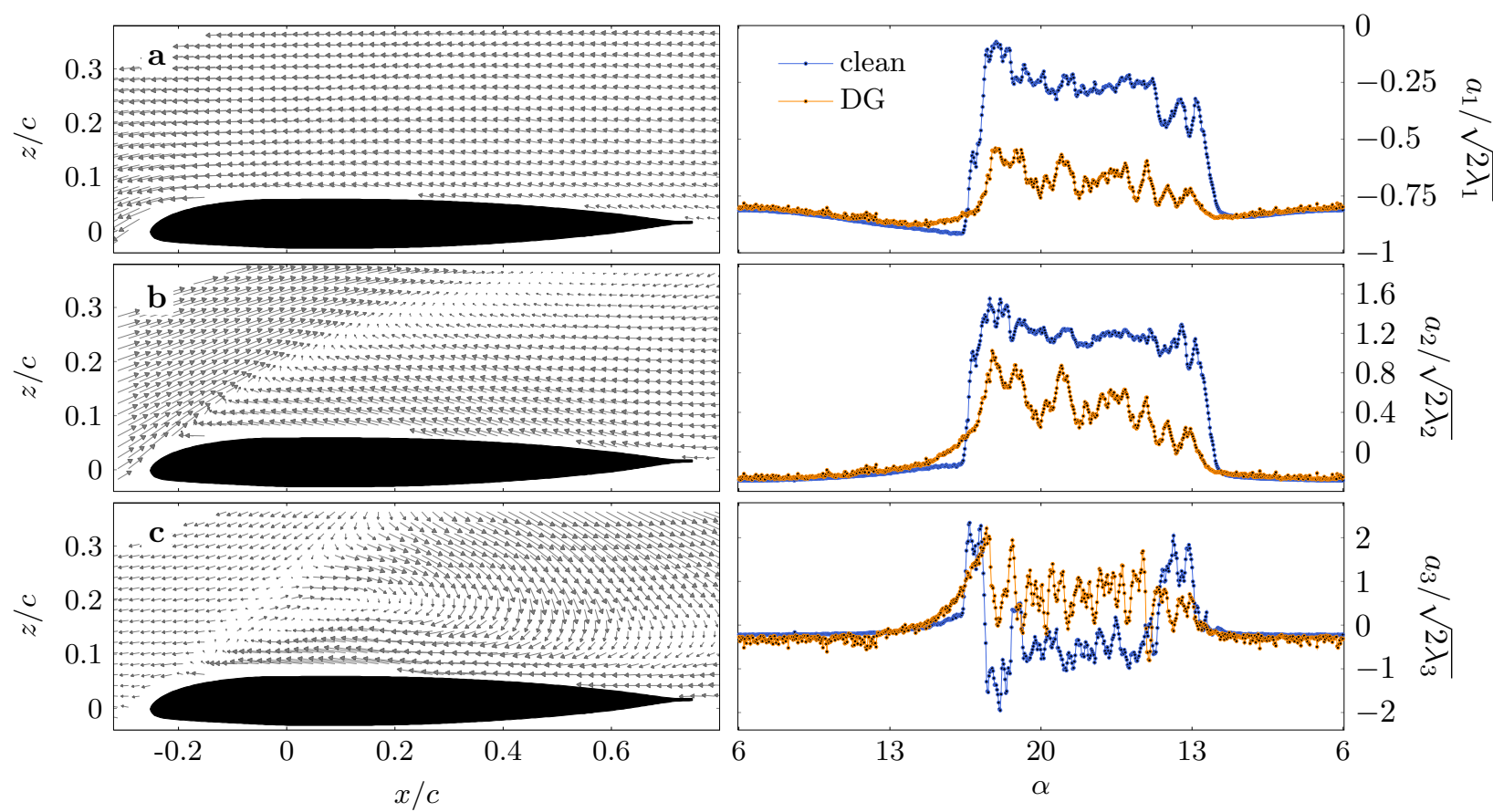

Figure 10. POD spatial eigenmodes (left column) of the velocity fields for the clean case and corresponding POD coefficients for the clean and the reference DG case. First row: mode 1, second row: mode 2 and third row: mode 3 at $\alpha=13 \pm 7^{\circ}, \mathbf{k}=\mathbf{0 . 0 4}$.

\section{III.B. Parameter Variation}

\section{III.B.1. Influence of the Shape, Size and Distribution of the Vortex Generators}

For static measurements at $\alpha \leq 14^{\circ}$ there is no measurable influence of the DGs for all configurations. If the angle of attack is further increased, distinct features of the different configurations were observed. The DGs with larger height $(h=0.18 \% \mathrm{c})$ and larger spacing $(s=6.66 \% \mathrm{c}$ and $s=10 \% \mathrm{c})$ could successfully delay stall by $2^{\circ}$ and more. Within the measured range of angles of attack, the shape played a minor role. In some cases of the small height $(h=0.108 \% \mathrm{c})$ and smaller spacing $(s=4 \% \mathrm{c}$ and $s=6.66 \% \mathrm{c})$ the DGs have a negative impact and the flow separated early. The shape of the DGs plays a more important role here and the circular DG was superior compared to the wedges. However, no clear correlation between shape, spacing and the stall process was found.

In summary, the larger height and larger spacing appears to delay stall more successfully, with some advantages to the spacing $s=6.66 \% \mathrm{c}$. The influence of the shape could not be resolved from the static measurements.

The analysis of the dynamic measurements showed that in addition to the reference case, other DG configurations have a positive influence on the airfoil performance. Figure 11 depicts the three 'best' configurations of each shape and the clean case. As observed for the static case, the larger height $h$ and larger spacing $s$ was more successful in reducing the hysteresis and pitching moment peak. While the differences in the lift are small, the variations in the pitching moment peak are significant. The best configuration in this case is the forward wedge ( $h=0.18 \% \mathrm{c}$ and $s=10 \% \mathrm{c})$, where the pitching moment peak was $53 \%$ lower, and the lift at $\alpha=16.5^{\circ} \downarrow 64 \%$ higher compared to the clean case. As observed for the reference DG case, stall does not occur as abrupt as for the clean case.

With regard to all dynamic measurements, the large height of $h=0.18 \% \mathrm{c}$ and the larger spacing $6.66 \% c \leq s \leq 10 \% c$ gives better results in terms of reducing the hysteresis effects and pitching moment peaks. While the lift coefficients are similar for all shapes at a given height and spacing, the pitch moment peaks and the reattachment processes differ significantly from shape to shape. The circular and the backward wedge configurations usually show very similar results, while the forward wedge configurations shows a larger difference. In most cases the circular and the backward wedge configuration produce a much lower pitching moment peak. However, the mentioned observations are not entirely consistent and in some cases, other configurations show a superior behavior. 

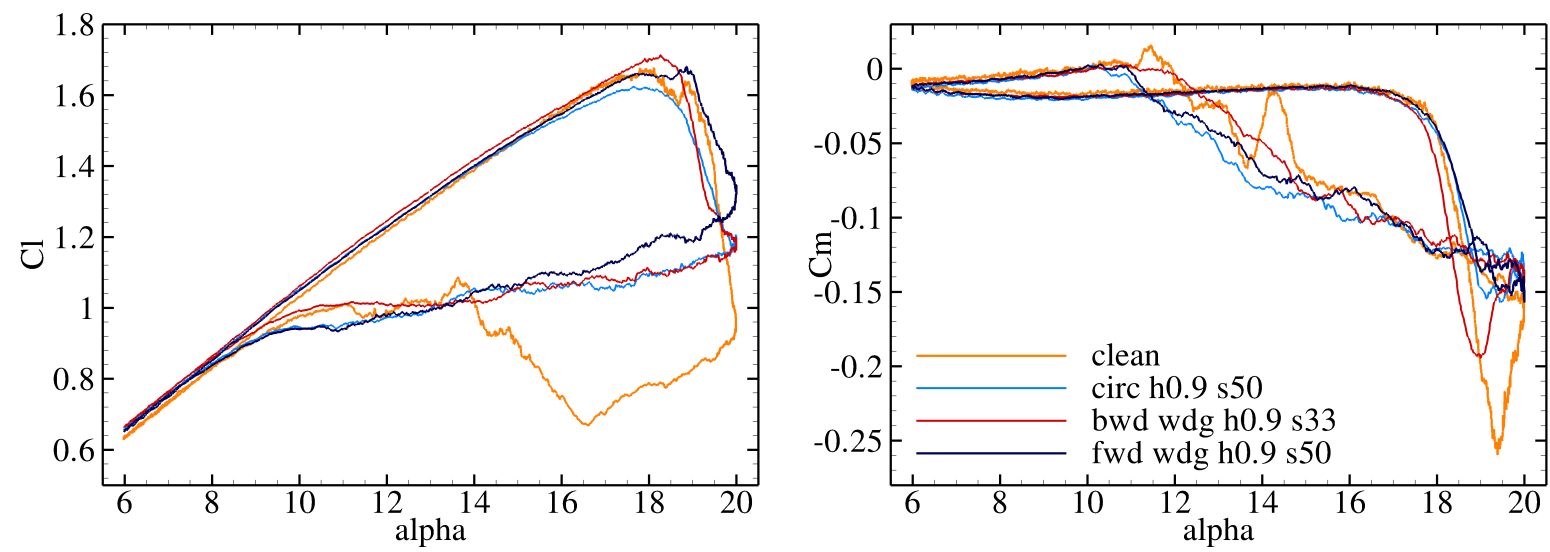

Figure 11. Time averaged dynamic lift and moment curves from pressure measurements plotted over angle of attack for clean and disturbance generator configuration $2, \mathbf{5}, 10$ at $\alpha=13 \pm 7^{\circ}, \mathbf{k}=\mathbf{0 . 0 4}$.

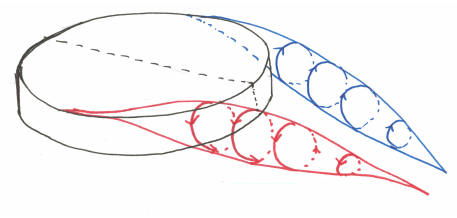

(a)

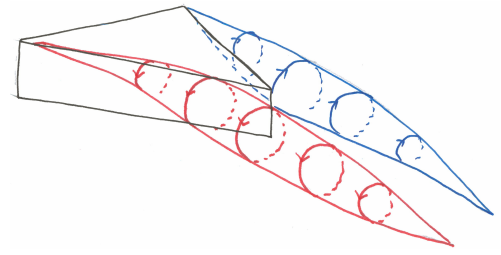

(b)

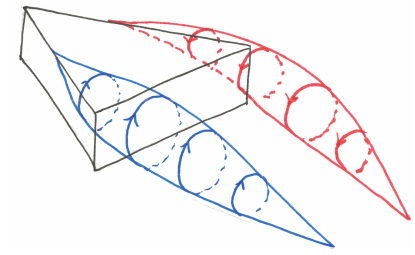

(c)

Figure 12. Sketches of the wake structure behind different DGs: a): circular, b) backward wedge, c) forward wedge.

An explanation for the differences between circular, backward and forward wedge configuration is given by the different wake structures of the DGs as shown in figure 12. While the circular and the backward wedge DG produce a pair of counter-rotating vortices that induce an upward velocity at the center the wake, the forward wedge DG produces a pair of vortices that rotate in the opposite sense. Pearcy ${ }^{25}$ applied the inviscid theory to predict the cross flow motion of co- and counter-rotating vortices as they convect downstream. He found that vortex pairs that induce an upward velocity at the center of the wake, move closer together as they travel downstream. In contrast, vortex pairs with the opposite sense of rotation will move away from each other. As these vortices get closer to vortices of the neighboring vortex generator, they stop their spanwise motion and the same situation as in the previous case is present, but at an older vortex age. Current investigations of the author ${ }^{18}$ focus on the interaction of the trailing vortices behind an array of DGs.

The influence of the DGs on the dynamic hysteresis loop of the moment coefficient can also be quantified by the aerodynamic damping, which is widely used as a measure of the moment stability. Negative damping causes high pitch link loads and blade torsion, which can limit a helicopter's flight envelope. If the value of the closed-loop integral $S$ over one cycle of the pitch moment loop is negative, negative damping is present. In order to find the damping coefficient $S^{*}$, the closed loop integral $S$ is non dimensionalized by the theoretical value of the integral. ${ }^{26}$ This damping coefficient has only physical meaning if analyzed for the forcing frequency (1/ref). Higher harmonics can be analyzed using the approach by Klein et al. ${ }^{27}$ where a discrete Fourier transformation (DFT) is performed in order to isolate the damping of the higher harmonics. The damping coefficients relative to the clean case of the first mode (1/ref) for selected DG configurations are summarized in table 3 . Even so the damping remains negative for all measured polars, the DG reduce the extent of the negative area under the moment-loop for most cases. At the forcing frequency (1/ref) damping is reduced up to $93 \%$ for the cases in table 3 . The DG generally reduce the hysteresis of the moment loop and therefore also the 'negative' area integrated with the closed loop integral. Only one configuration (IV-1) has a lower damping than the clean case. This is due to the reduced pitching moment peak that does not allow a 'positive' contribution to the damping coefficients for high angles of attack as in the clean case. A similar effect can be observed in figure 11 for the clean and the circular case No. $2(h=0.18 \% \mathrm{c}, s=10 \% \mathrm{c})$. The higher harmonics from $2 /$ ref to $6 /$ ref show a strong variation with mostly positive values. However, 


\begin{tabular}{|c|c|c|c|c|c|c|}
\hline case & $\alpha_{0}$ & $\hat{\alpha}$ & $\mathbf{k}$ & $S_{1}^{*}$ & $\Delta S_{1}^{*}$ & $\Delta \mathrm{cm}_{\min }$ \\
\hline & {$\left[^{\circ}\right]$} & $\left.{ }^{\circ}\right]$ & {$[-]$} & {$[-]$} & {$[\%]$} & {$[\%]$} \\
\hline \hline II-clean & 13 & 5 & 0.1 & -4.2 & - & - \\
\hline II-1 & 13 & 5 & 0.1 & -0.3 & 93 & 6 \\
\hline II-2 & 13 & 5 & 0.1 & -1.3 & 69 & 26 \\
\hline II-5 & 13 & 5 & 0.1 & -2.7 & 36 & 20 \\
\hline II-6 & 13 & 5 & 0.1 & -2.1 & 50 & 21 \\
\hline II-9 & 13 & 5 & 0.1 & -1.1 & 74 & -21 \\
\hline II-10 & 13 & 5 & 0.1 & -3.8 & 10 & -22 \\
\hline IV-clean & 13 & 7 & 0.04 & -1.9 & - & - \\
\hline IV-1 & 13 & 7 & 0.04 & -2.6 & -37 & 30 \\
\hline IV-2 & 13 & 7 & 0.04 & -1.7 & 11 & 39 \\
\hline IV-5 & 13 & 7 & 0.04 & -1.9 & 0 & 32 \\
\hline IV-6 & 13 & 7 & 0.04 & -1.5 & 21 & 39 \\
\hline IV-9 & 13 & 7 & 0.04 & -0.7 & 63 & 21 \\
\hline IV-10 & 13 & 7 & 0.04 & -1.5 & 22 & 6 \\
\hline
\end{tabular}

Table 3. Damping coefficients of selected DG configurations.

absolute values are very small for all cases and hence the influence is negligible.

\section{III.B.2. Influence of transition}

To investigate the effectiveness of the DG with regard to the state of the boundary layer, several cases with boundary layer tripping have been measured. While the boundary layer at the position of the DGs $(x / c \approx$ $1.2 \%$ chord) is usually laminar, it has been tripped to turbulent at $3 \%$ chord for these cases. The height of the 'transition disc' tripping strip was calculated with a modified version of the method developed by Van Driest et $a l^{28}$

$$
h_{t r}=\sqrt{1050\left(1+\frac{\gamma+1}{2} M_{\text {delta }}^{2}\right) \frac{c \delta^{*}}{R e}}
$$

Where $M_{\text {delta }}$ is the Mach number at the outside of the boundary layer and $\delta^{*}$ is the displacement thickness at the desired transition location. Both $M_{\text {delta }}$ and $\delta^{*}$ were extracted from MSES ${ }^{29}$ calculations. In the present case, a height of $h_{t r}=285 \mu \mathrm{m}$ for the upper, and $h_{t r}=200 \mu \mathrm{m}$ for the lower surface was calculated. The results of the measurements show only small differences between the cases with boundary layer tripping and without, for the DGs and the clean cases. Even so, the DGs certainly trigger transition after the intermittency length due to their height far above the critical height, so the aerodynamic improvements seen are not a result of simple boundary layer tripping. The DG cases without the transition strip still show the large improvements compared to the clean case with the transition strip.

The small influence of the boundary layer type can be explained by the low thickness of the boundary layer, compared to the DG height at the position of the DG. Static MSES calculations give a minimum ratio of $h / \delta=15$ for the large DGs and $h / \delta=9$ for the small DGs. According to flat plate theory, the turbulent boundary layer at the DG position is approximately twice as high as the laminar counterpart and therefore the DG height is still much higher than the boundary layer and it emerges into the free stream. Investigations of the wake behind circular DGs ${ }^{18}$ showed that for $h / \delta \leq 1$, the wake structure is similar for all cases. Only once the DG is submerged into the boundary layer, the structures of the wake change.

\section{Conclusion}

Leading edge devices have been found to significantly improve the performance of an OA209 airfoil under dynamic stall conditions. In order to better understand the principles of operation, a pitching OA209 airfoil with passive disturbance generators has been investigated experimentally in a wind tunnel by means of 
time resolved PIV and pressure measurements. Several different leading edge device geometries, sizes and spacing have been applied in order to study their influence on the flow. As for the circular shape (DGs) in previous experiments, most configurations of the disturbance generators show large improvements for static and dynamic measurements. Especially for dynamic cases the DG reduced successfully the negative pitching moment peak of up to $53 \%$ and the hysteresis effects. While the previous experiments were based on pressure measurements, the temporal and spatial development of the flow field above the pressure sensors could be measured in the present work. With this information vortices could be detected and tracked revealing a clear difference between the baseline cases and the configurations with disturbance generators. While the flow around the clean airfoil separates abruptly near the leading edge (leading edge stall) and the vortices convect far away from the airfoil, the flow separation is delayed by the DG and vortices stay closer to the wall. The POD analysis over one cycle showed that during stall, the coherent structures in the flow field are weaker for the DG cases, indicating weaker vortices.

The DGs also have a positive influence on the aerodynamic damping for the $1 /$ ref signal and higher harmonics. The damping coefficient could be increased by up to $93 \%$. In general larger size and larger spacing of the DG works better with a height of $(h=0.18 \% \mathrm{c})$ and a spacing $(6.66 \% \mathrm{c} \leq s \leq 10 \% \mathrm{c})$ being optimal. Also the circular and backward wedge perform better than the forward wedge shape, which can be attributed to the different wake structure.

In general, the DG configurations found to be successful, have a positive influence for all forcing motions. However, the magnitude of the changes strongly depend on the configuration and the dynamic stall case. To date no clear correlation between the airfoil motion and the influence of the DG was found, but this is the subject of ongoing investigations. A second study is focusing on the exact wake structure behind different DG configurations.

\section{Acknowledgments}

This work is part of the DLR and ONERA joint project 'Advanced Simulation and Control of Dynamic Stall' (SIMCOS). We would like to thank Jean-Michel Deluc, Frédéric David, Thibault Joret and Yannik Amosse for thoroughly acquiring the data and preparing the model and the test rig. We also thank Philippe Loiret, manager of the F2 wind tunnel, for the excellent cooperation.

\section{References}

${ }^{1}$ McCroskey, W., "The Phenomenon of Dynamic Stall," NASA TM-81264, 1981. 1977.

${ }^{2}$ Carr, L., "Analysis of the Development of Dynamic Stall Based on Oscillating Airfoil Experiments," NASA TN-8382,

${ }^{3}$ Leishman, J., "Dynamic Stall Experiments on the NACA 23012 Aerofoil," Experiments in Fluids, Vol. 9, No. 1, 1990, pp. $49-58$.

${ }^{4}$ Mulleners, K., Henning, A., Mai, H., and Raffel, M., "Investigation of the Unsteady Flow Development over a Pitching Airfoil by Means of TR-PIV," AIAA Paper 2009-3504, 2009.

${ }^{5}$ Geissler, W. and M., R., "Dynamic Stall Control by Airfoil Deformation," 19. European Rotorcraft Forum, Vol. $1,1993$.

${ }^{6}$ Chandrasekhara, M., Wilder, M., and Carr, L., "Unsteady Stall Control Using Dynamically Deforming Airfoils," AIAA Journal, Vol. 36, No. 10, 1998, pp. 1792-1800.

${ }^{7}$ Carr, L., Chandrashekara, M., Wilder, M., and Noonan, K., "The Effect of Compressibility on Suppression of Dynamic Stall Using a Slotted Airfoil," AIAA Paper 1998-332, 1998.

${ }^{8}$ Carr, L. and McAlister, K., "The Effect of a Leading-Edge Slat on the Dynamic Stall of an Oscillating Airfoil," AIAA Paper 1983-2533, 1983.

${ }^{9}$ Greenblatt, D. and Wygnanski, I., "Dynamic Stall Control by Periodic Excitation, Part1: NACA 0015 Parametric Studies," Journal of Aircraft, Vol. 38, No. 3, 2001, pp. 430-438.

${ }^{10}$ Post, M. and Corke, T., "Separation Control Using Plsama Actuators: Dynamic Stall Vortex Control on Oscillating Airfoil," AIAA Journal, Vol. 44, No. 12, 2004, pp. 3125-3135.

${ }^{11}$ Martin, P., Wilson, J., Wong, J. B. T., Moulton, M., and McVeigh, M., "Passive Control of Compressible Dynamic Stall," AIAA Paper 2008-7506, 2008.

${ }^{12}$ Geissler, W., Dietz, G., Mai, H., Bosbach, J., and H., R., "Dynamic Stall and its Passive Control Investigations on the OA209 Airfoil Section," 31. European Rotorcraft Forum, 2005.

${ }^{13}$ Dietz, G., Mai, H., and Geissler, W., "Auftriebsfläche mit verbessertem Ablöseverhalten bei stark veränderlichem Anstellwinkel," German Patent, , No. DE102005018427, 2005.

${ }^{14}$ Dietz, G., Mai, H., and Geissler, W., "Lifting surface with improved separation behaviour under strongly variable angle of incidence," December 2008.

${ }^{15}$ Mai, H., Dietz, G., Geissler, W., Richter, K., Bosbach, J., Richard, H., and de Groot, K., "Dynamic Stall Control by Leading Edge Vortex Generators," American Helicopter Society, Vol. 62, 2006. 
${ }^{16}$ Heine, B., Mulleners, K., and Gardner, A., "On the Effects of Leading Edge Vortex Generators on an OA209 Airfoil," 10th ONERA-DLR Aerospace Symposium ODAS Berlin, 2009.

${ }^{17}$ Heine, B., Schwermer, T., and Raffel, M., "The Effect of Vortex Generators on the Flow around a Circular Cylinder," 15th Int Symp on Applications of Laser Techniques to Fluid Mechanics, Lisbon, 2010.

${ }^{18}$ Heine, B., Schanz, D., Schröder, A., Dierksheide, U., and Raffel, M., "Investigation of the Wake of Low Aspect Ratio Cylinders by Tomographic PIV," 9th International Symphosium on particle image velocimetry - PIV11, 2011.

19 "Experimental Study of Dynamic Stall Control using Deployable Leading-Edge Vortex Generators," Proceedings of the American Helicopter Society $67^{\text {th }}$ Annual Forum, American Helicopter Society International, Virginia Beach, VA, USA, 3-5 May 2011.

${ }^{20}$ Raffel, M., Willert, C., Wereley, S., and Kompenhans, J., Particle Image Velocimetry - a Practical Guide, Springer, 2007.

${ }^{21}$ Graftieaux, L., Michard, M., and Grosjean, N., "Combining PIV, POD and vortex identification algorithms for the study of unsteady turbulent swirling flows," Measurement Science and Technology, Vol. 12, No. 9, 2001, pp. 1422.

${ }^{22}$ Mulleners, K. and Raffel, M., "The Onset of Dynamic Stall Revisited," Exp. Fluids, 2011, in print.

${ }^{23}$ Sirovich, L., "Turbulence and the Dynamics of Coherent Structures Part1: Coherent Structures," Quarterly of Applied Mathematics, Vol. 45, No. 3, 1987, pp. 561-571.

${ }^{24}$ Mulleners, K. and Raffel, M., "The Onset of Dynamic Stall: a Time-resolved Approach," Proceedings of the $36^{\text {th }}$ European Rotorcraft Forum, Paris, France, September 07-09 2010. 1961.

${ }^{25}$ Pearcey, H., Boundary layer and flow control, its principle and applications, Vol 2, Pergamon Press, Oxford, England,

${ }^{26}$ Theodorsen, T., "General theory of aerodynamic instability and the mechanism of flutter," NACA Report, Vol. 496, 1969.

${ }^{27}$ Klein, A., Richter, K., Altmikus, A., Lutz, T., and Krämer, E., "Unsteady Criteria for Rotor Blade Airfoil Design," 35th European Rotorcraft Forum, 2009.

${ }^{28}$ Van Driest, E. and Blumer, C., "Effect of roughness on transition in supersonic flow," AGARD Report, Vol. 255, 1960.

${ }^{29}$ Drela, M. and Giles, M., "Viscous-Inviscid Analysis of Transonic and Low Reynolds Number Airfoils," AIAA Journal, Vol. 25, No. 10, 1987. 